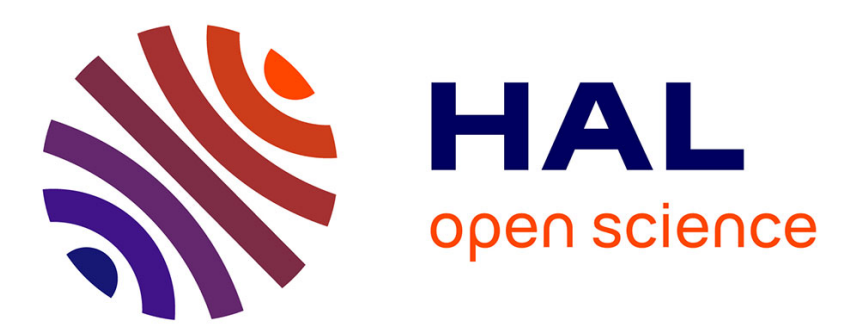

\title{
Diffraction Stress Analysis Using Direction Dependent Grain-Interaction Models
}

\author{
U. Welzel, Sylvain Fréour, A. Kumar, E. J. Mittemeijer
}

\section{To cite this version:}

U. Welzel, Sylvain Fréour, A. Kumar, E. J. Mittemeijer. Diffraction Stress Analysis Using Direction Dependent Grain-Interaction Models. Materials Science Forum, 2005, 490-491, pp.7-12. 10.4028/www.scientific.net/MSF.490-491.7 . hal-01006746

\section{HAL Id: hal-01006746 \\ https://hal.science/hal-01006746}

Submitted on 11 Jan 2019

HAL is a multi-disciplinary open access archive for the deposit and dissemination of scientific research documents, whether they are published or not. The documents may come from teaching and research institutions in France or abroad, or from public or private research centers.
L'archive ouverte pluridisciplinaire HAL, est destinée au dépôt et à la diffusion de documents scientifiques de niveau recherche, publiés ou non, émanant des établissements d'enseignement et de recherche français ou étrangers, des laboratoires publics ou privés. 


\title{
Diffraction Stress Analysis Using Direction-Dependent Grain-Interaction Models
}

\author{
U. Welzel. S. Fréour, A. Kumar and E. J. Mittemeijer \\ Max Planck Institute for Metals Research, Heisenbergstr. 3, D-70569 Stuttgart, Germany
}

Keywords: diffraction stress analysis, grain interaction, surface anisotropy, grain-shape texture

\begin{abstract}
Recently, so-called direction-dependent elastic grain-interaction models have been developed, which are capable of considering the effects of surface anisotropy or of a grain-shape (morphological) texture on the mechanical elastic constants and diffraction (X-ray) stress factors of polycrystals. The notion 'direction-dependent grain-interaction' signifies that different graininteraction constraints prevail along different directions in the polycrystal. This work summarizes recent developments and presents a comparison of direction-dependent elastic grain-interaction models.
\end{abstract}

\section{Introduction}

Elastic grain-interaction models describe the distribution of stresses and strains over the crystallographically differently oriented grains in a polycrystal. They can be employed for the calculation of mechanical elastic constants (relating mechanical strains to mechanical stresses) and diffraction elastic constants (diffraction (X-ray) stress factors for mechanically elastically anisotropic samples, relating (diffraction) lattice strains to mechanical stresses) from single-crystal elastic data [1-3].

Usually, isotropic grain-interaction models, as for example the Reuss [4], Voigt [5], Neerfeld-Hill [6,7] or Eshelby-Kröner [8,9] models, are considered. In such isotropic models, the same grain-interaction assumptions are adopted for all directions in the polycrystal. The Voigt and Reuss models involve extreme grain-interaction assumptions: either the strain tensor (Voigt model) or the stress tensor (Reuss model) for each crystallite is assumed to be equal to the mechanical strain or stress tensor, respectively; they are incompatible with physical reality because of the corresponding stress, respectively strain discontinuities at the grain boundaries. It was demonstrated by Hill [7] that the Voigt and Reuss models set bounds for the mechanical elastic constants. Further, Neerfeld [6] and Hill [7] found on an empirical basis that the arithmetic (or geometric, Hill, [7]) averages of X-ray [6] and macroscopic [6,7] elastic constants calculated according to the models of Voigt and Reuss are generally in good agreement with experimental data. Close to these values are also the results of the (mathematically more tedious) Eshelby-Kröner model.

Devised for bulk specimens with an isotropic microstructure, the above mentioned models imply mechanical elastic isotropy for crystallographically untextured polycrystals. However, a polycrystal cannot generally be considered as being mechanically elastically isotropic, even in the absence of crystallographic texture.

Thin films or surface layers of bulk-polycrystals, as examples, can exhibit mechanically elastically only transverse isotropy, owing to their microstructure and reduced dimensionality [10,11]. Thus, anisotropic grain interaction occurs. In a (columnar) thin film (or the surface layer of a bulk polycrystal), each crystallite is surrounded by neighbouring crystallites in only two dimensions. Thus, the grain-interaction perpendicular to the surface can be different from the grain interaction parallel to the surface [10,12-14] (see also Stickforth [11], who introduced the notion 'surface-anisotropy' of bulk polycrystals).

Polycrystals with a grain-shape (morphological) texture also are macroscopically elastically anisotropic. The effect of a morphological texture on the elastic behaviour can be considered by employing an extension of the Eshelby-Kröner grain-interaction model [15,16]; see, in particular, 
Koch et al. [17]). To this end, a calculation scheme involving ellipsoidal grains (inclusions) with their principal directions aligned along common axes in the specimen frame of reference, where the $S_{3}$-axis is perpendicular to the sample surface, can be adopted (i.e., an ideal grain-shape texture occurs; for a detailed discussion, see [17]), whereas spherical grains (inclusions) are considered in the traditional Eshelby-Kröner model.

\section{Mechanical Elastic Constants and X-Ray Stress Factors}

In thin films, plane, rotationally symmetric states of mechanical stress/strain (mechanical averages of tensors are indicated by brackets ' $<>$ ') are frequently met:

$$
\left\langle\varepsilon^{S}\right\rangle=\left(\begin{array}{ccc}
\varepsilon_{\square}^{S} & 0 & 0 \\
0 & \varepsilon_{\square}^{S} & 0 \\
0 & 0 & \varepsilon_{\perp}^{S}
\end{array}\right), \quad\left\langle\sigma^{S}\right\rangle=\left(\begin{array}{ccc}
\sigma_{\square}^{S} & 0 & 0 \\
0 & \sigma_{\square}^{S} & 0 \\
0 & 0 & 0
\end{array}\right) .
$$

The superscript $S$ indicates that a tensor is expressed in the specimen frame of reference $S$. Under these loading conditions, which will be considered for the Vook-Witt and the inverse Vook-Witt models discussed below, two mechanical elastic constants A and B suffice, as only three independent non-zero stress and strain tensor components occur:

$$
\begin{aligned}
& \varepsilon_{\square}^{S}=\mathrm{A} \sigma_{\square}^{S}, \\
& \varepsilon_{\perp}^{S}=\mathrm{B} \sigma_{\square}^{S} .
\end{aligned}
$$

For macroscopically elastically anisotropic specimens, the $\varphi, \psi$ and $h k l$-dependent diffraction (Xray) stress factors $F_{i j}(\psi, \varphi, h k l)$ relate the diffraction strain $\varepsilon_{\varphi, \psi}^{h k l}$, measured for an $h k l$ reflection at a specimen tilt angle $\psi$ and rotation angle $\varphi$, to the mechanical stress tensor expressed in the specimen frame of reference $\left\langle\sigma^{S}\right\rangle[1-3]$ :

$$
\varepsilon_{\varphi, \psi}^{h k l}=F_{i j}(\psi, \varphi, h k l)\left\langle\sigma_{i j}^{S}\right\rangle .
$$

Note that the $F_{i j}(\psi, \varphi, h k l)$ are not components of a tensor since they relate the lattice strain (a number) to the stress tensor (expressed in the $S$-system). For the case of transverse elastic isotropy and under a plane, rotationally symmetric state of stress, the diffraction strain is independent of the angle $\varphi$, thus, $\varphi$ can be arbitrarily set to zero (or any other value) for the X-ray stress factors and $\varphi$ as an index can be suppressed for the diffraction strain, so that Eq. (5) can be simplified:

$$
\varepsilon_{\psi}^{h k l}=\left(F_{11}(\psi, 0, h k l)+F_{22}(\psi, 0, h k l)\right) \sigma_{\square}^{S} .
$$

Usually, the lattice strain is plotted versus $\sin ^{2} \psi$ in a so-called $\sin ^{2} \psi$-plot.

\section{Thin Films / Surface Anisotropy}

In (columnar) thin films and surface layers of bulk polycrystals, neighbouring grains surround a grain in only two dimensions. Grain-interaction assumptions taking this into account can be formulated as follows ([10], see also Vook \& Witt [18]; a mechanical loading state as given by Eqs. (1) and (2) is considered): (i) in the plane of the film, the strain is rotationally symmetric and (ii) equal for all crystallites and (iii) the stresses perpendicular to the layer are zero for all crystallites, i.e. the crystallites can deform freely in this direction. The above formulated grain-interaction assumptions allow the calculation of the mechanical elastic constants A and B and the (sum of two, see Eq. (6)) diffraction stress factors [10, 12-14].

Extreme grain-interaction assumptions (e.g. the stresses perpendicular to the layer are zero for all crystallites) are adopted in the Vook-Witt (VW) model. In terms of the bulk models it could be said that, a Voigt behaviour is attributed to the in-plane directions, whereas a Reuss behaviour is 
attributed to the direction perpendicular to the film surface. Thus, the VW model will generally be incompatible with the true elastic behaviour of a polycrystal. To overcome this problem, the inverse Vook-Witt (iVW) model, required for the construction of an effective grain-interaction model, has been proposed by Welzel et al. [13,14]. The grain-interaction assumptions for the iVW model are as follows: (i) the in-plane stress is rotationally symmetric and (ii) equal for all crystallites and (iii) the strain perpendicular to the film surface is equal for all crystallites. As in the VW model, the above formulated grain-interaction assumptions allow the calculation of the mechanical elastic constants A and B and the (sum of two, see Eq. (6)) diffraction stress factors [13,14].

In an effective grain-interaction model, the mechanical and diffraction elastic constants are calculated as arithmetic averages of the elastic constants obtained from a set of extreme graininteraction models. For the following discussion such an average of (extreme) grain-interaction models will be called an effective grain-interaction model. A well known example for an effective grain-interaction is the Neerfeld-Hill model (cf. Introduction). The background of any averaging of (extreme) grain-interaction models to describe physical reality could be described as follows: A real sample is conceived to be constituted from separate volume fractions of crystallites, each of which obeys a certain type of grain-interaction.

The need for introducing the iVW model can be understood as follows: As compared to bulk materials where all directions for grain interaction can be equivalent, thin films posses two principal directions exhibiting possibly different types of grain interactions: the in-plane direction (all inplane directions are equivalent) and the direction perpendicular to the surface of the film. The number of grain-interaction models of extreme types of grain-interaction assumptions is two for bulk materials (Reuss and Voigt). Consequently, four types of extreme grain-interaction models then can be formulated for columnar thin films, as two principal directions, each with two extreme grain-interactions, occur. These extreme grain-interaction models are the Reuss, the Voigt, the Vook-Witt and the inverse Vook-Witt models.

The VW model and the iVW model imply that a polycrystal is macroscopically elastically anisotropic (transversely isotropic). Of course, this mechanical anisotropy cannot be revealed by the two elastic constants A and B (cf. Table 1 for an example). However, the occurrence of non-linear $\sin ^{2} \psi$-plots for crystallographically untextured polycrystals subjected to a plane, rotationally symmetric state of stress (see Eq. (2)) indicates that indeed mechanical elastic anisotropy does occur; see Fig. 1, where pronounced curvature in the $\sin ^{2} \psi$-plots can be observed. Experimental investigations of direction-dependent graininteraction in thin films have been conducted by van Leeuwen et al. [10] and Welzel et al. [13,14].

Table 1: Mechanical elastic constants A and B of a crystallographically untextured copper polycrystal (singlecrystal elastic data from [19]) according to the Reuss (R), Voigt (V), Vook-Witt (VW) and inverse Vook-Witt (iVW) models.

\begin{tabular}{|c|c|c|c|c|}
\hline $\mathrm{Cu}$ & $\mathrm{R}$ & $\mathrm{V}$ & $\mathrm{VW}$ & iVW \\
\hline $\mathrm{A}\left(\mathrm{TPa}^{-1}\right)$ & 5.76 & 4.65 & 5.19 & 4.91 \\
\hline $\mathrm{B}\left(\mathrm{TPa}^{-1}\right)$ & -6.72 & -4.50 & -5.59 & -5.03 \\
\hline
\end{tabular}

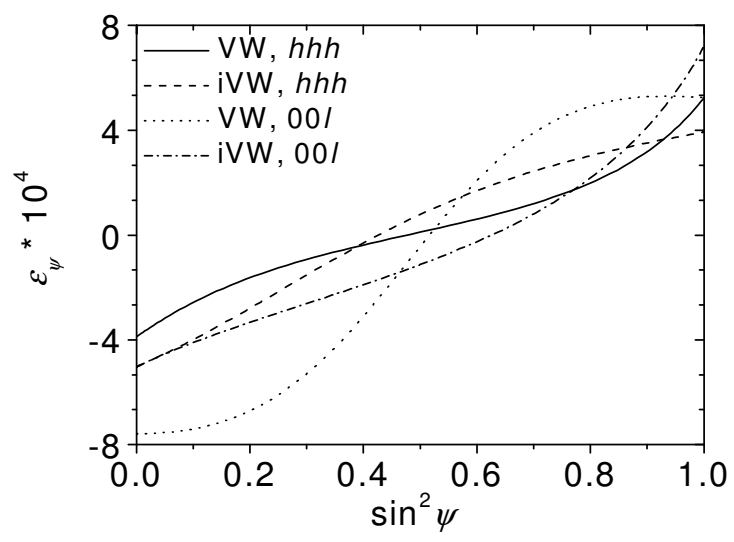

Figure 1: $\sin ^{2} \psi$-plots for the $h h h$ and $00 l$ reflections of a crystallographically untextured copper polycrystal subjected to a plane-rotationally symmetric state of stress (100MPa) according to the Vook-Witt (VW) and inverse Vook-Witt (iVW) models (single-crystal elastic data from [19]). Note that a plot of $e_{y}$ versus $\sin ^{2} \psi$ is equivalent to a plot of $F_{11}+F_{22}$ versus $\sin ^{2} \psi$ (cf. equation (6)). 


\section{Grain Shape (Morphological) Texture}

In order to calculate the elastic constants of a polycrystal from single-crystal elastic data in the Eshelby-Kröner (EK) model, the crystallites surrounding an individual grain (inclusion) in a polycrystal are conceived as an elastically homogenous matrix with the elastic properties of the entire polycrystal $[8,9]$.

Traditionally, a spherical shape of the inclusions is considered [9]. The effect of a grain-shape (morphological) texture on mechanical and diffraction elastic constants can be considered in the EK model by considering ellipsoidal inclusions with their principal axes aligned along common directions in the specimen frame of reference. The shape of the crystallites can be described by a shape parameter $h$, which is defined as the ratio of the principal axis of the ellipsoid in the $z-$ direction $\left(a_{3}\right)$ of the specimen frame of reference and the principal axes of the ellipsoid in the $\mathrm{x}$ direction $\left(a_{1}\right)$ and the y-direction $\left(a_{2}\right)$ in the specimen frame of reference, respectively:

$$
h=\frac{a_{3}}{a_{1}}=\frac{a_{3}}{a_{2}}
$$

Thus, the considered ellipsoids exhibit rotational symmetry with respect to the surface normal of the specimen. Only an ideal grain-shape, morphological texture is considered in the following, as only in this case unique mechanical elastic constants and X-ray stress factors can be calculated employing the EK model (for a more detailed discussion of the effect of a non-ideal morphological texture, see [17]).

Examples for mechanical and diffraction elastic constants calculated for grain aspect ratios $0.01 \leq \eta \leq 100$ are given in Fig. 2. For $h^{1} 1$ (i.e., non-spherical grains), macroscopic elastic anisotropy occurs. This is revealed by the occurrence of curved $\sin ^{2} \psi$-plots (as this model allows the calculation of all components of the mechanical stiffness tensor, the mechanical elastic anisotropy, transverse isotropy in this case, could also be deduced from an inspection of this tensor; see [17] for examples). It has to be stressed that it is the preferential alignment of non-spherical grains that induces the macroscopic elastic anisotropy. Obviously, a polycrystal consisting of non-spherical grains with a random distribution of their (shape) orientations will be macroscopically elastically isotropic, as no preferred morphological direction occurs.

The mechanical elastic constants exhibit a weak dependence on the grain-aspect ratio $h$ whereas the effect on the diffraction elastic constants is more pronounced.

\section{Comparison of the Direction-Dependent Elastic Grain-Interaction Models}

At a first glance, the occurrence of surface anisotropy and the occurrence of a morphological texture bear no relation and thus, similarities among the elastic constants calculated with the VW/iVW and the EK models cannot be expected. However, upon comparing Fig. 1 with Fig. 2a, it is striking that similarities in the $\sin ^{2} \psi$-plots do occur.

In Fig. 2b, the mechanical elastic constants A and B (cf. Eqs. (3) and (4)) calculated employing the EK model are shown as functions of the grain-aspect ratio $h$ (line with open circles). For comparison, the values of $\mathrm{A}$ and $\mathrm{B}$ have been indicated also for the VW, the iVW and the Reuss and Voigt models. It can be observed that the values of A and B calculated according to the EK model fall between the corresponding VW and iVW values. As the grain shape approaches the limit of a flat disc $(h \circledast 0)$, A and B tend towards the corresponding VW values, whereas in the limit of needle-like grains $(h \circledast \exists)$, A and B tend towards the corresponding iVW values. Hence, the similarities among the different models revealed by the $\sin ^{2} \psi$-plots also hold for the mechanical elastic constants A and B. These observations may be understood as follows. 


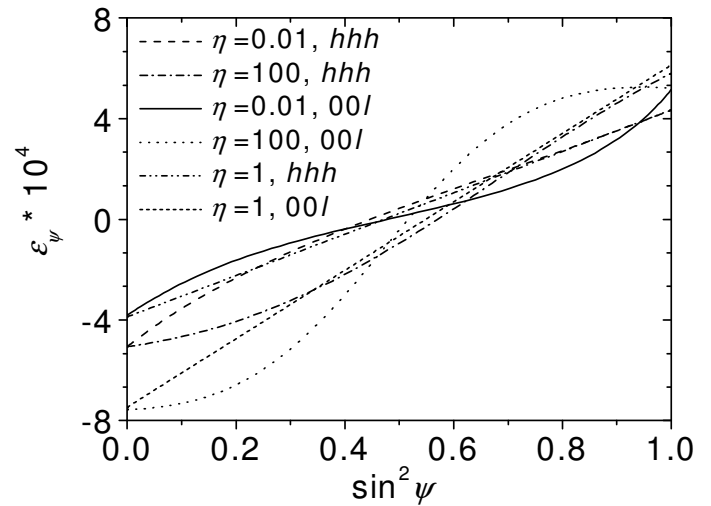

Figure 2a: $\sin ^{2} \psi$-plots for the $h h h$ and $00 l$ reflections of a crystallographically untextured copper polycrystal subjected to a plane-rotationally symmetric state of stress (100MPa) according to the EK model involving different ellipsoidal crystallite morphologies (singlecrystal compliances from [19]). Note that a plot of $e_{y}$ versus $\sin ^{2} \psi$ is equivalent to a plot of $F_{11}+F_{22}$ versus $\sin ^{2} \psi$ (cf. Eq. (6))
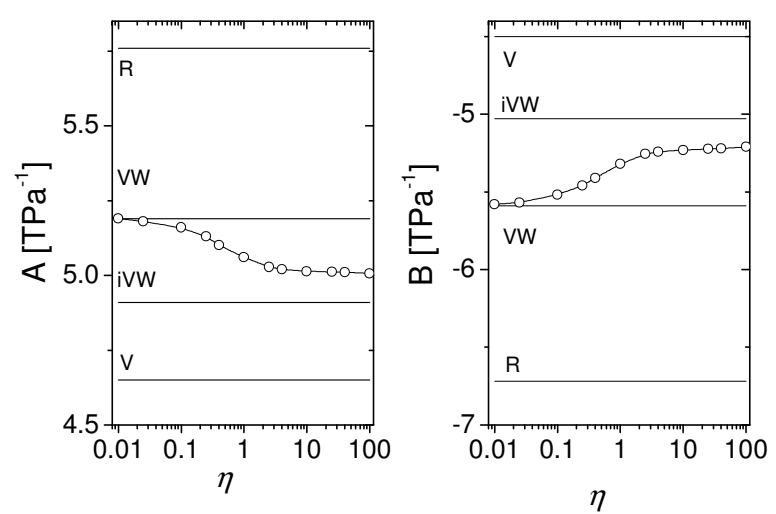

Figure 2b: Mechanical elastic constants A and B of a crystallographically untextured copper polycrystal according to the Vook-Witt (VW), inverse Vook-Witt (iVW) and the EK model as a function of the grain aspect ratio $h$ (line with open circles). For comparison, the values for A and B according to the Reuss (R) and Voigt (V) models have also been indicated (singlecrystal compliances from [19]).

In the case of flat-disc shaped grains (i.e., $\eta \rightarrow 0$ ) with the disc faces parallel to the surface of the specimen, a polycrystal could be conceived as a stack of flat disc-shaped grains extending throughout the entire width of the polycrystal. If such a polycrystal is subjected to a plane, rotationally symmetric mechanical strain parallel to the disc interfaces, all discs (grains) will experience the same in-plane strain. Due to mechanical equilibrium conditions, they will experience the same stress $(\stackrel{\circ}{0})$ perpendicular to the interfaces: the 'grain-interaction assumptions' are thus of the Vook-Witt type.

In the case of needle-shaped grains (i.e., $\eta \rightarrow \infty$ ), a polycrystal could be conceived as an assembly of needle-shaped grains with the needle axes perpendicular to the surface of the specimen. If such a polycrystal is subjected to a plane, rotationally symmetric mechanical strain perpendicular to the needle axes and if one requires that the outer contour of the assembly does not change, all needles will experience the same in-plane stress in order to satisfy the mechanical equilibrium conditions. Due to the requirement of surface contour-coherence, the grains will experience the same strain along the needle axes: the 'grain-interaction assumptions' are thus of the inverse Vook-Witt type. It is possible to confirm the here observed similarities by analytical simplifications of certain tensors involved in the calculations in the Eshelby-Kröner model for the limits $h \circledast 0$ and $h \circledast$ [20].

\section{Conclusions}

- Thin films can exhibit mechanical, macroscopic elastic anisotropy also in the absence of crystallographic texture. The elastic grain interaction of such thin films can be modelled employing an effective grain-interaction model combining the extreme Reuss, Voigt, Vook-Witt and inverse Vook-Witt models.

- Polycrystals with a grain-shape (morphological) texture are mechanically elastically anisotropic. Their elastic grain interaction can be modelled employing an extension of the traditional Eshelby-Kröner model; whereas spherical grains (inclusions) are considered in the traditional Eshelby-Kröner model, a calculation of mechanical elastic constants and diffraction stress factors is also possible considering ellipsoidal grains with their principal directions aligned along common axes in the specimen frame of reference. 
- For extreme grain morphologies (flat discs and needles), the grain-interaction in the EshelbyKröner model exhibits similarities with the grain interaction in the Vook-Witt and inverse Vook-Witt models, respectively. Even though the Vook-Witt and inverse Vook-Witt models have been developed in order to deal with the effect of elastic surface anisotropy of bulk polycrystals and thin films, they are thus capable of modelling the effect of an ideal morphological texture on the elastic grain interaction.

\section{References}

[1] V. Hauk, (editor): Structural and residual stress analysis by nondestructive methods (Elsevier, Amsterdam 1997).

[2] U. Welzel, J. Ligot, P. Lamparter, A.C. Vermeulen and E.J. Mittemeijer: submitted to J. Appl. Cryst.

[3] U.Welzel and E.J. Mittemeijer: J. Appl. Phys. 93 (2003), p. 9001

[4] A. Reuss: Zeitschrift für angewandte Mathematik und Mechanik 9 (1929), p. 49

[5] W. Voigt: Lehrbuch der Kristallphysik (Teubner, Leipzig-Berlin: 1910).

[6] H. Neerfeld: Mitt. K.-Wilh.-Inst. Eisenforschg. 24 (1942), p. 61

[7] R.Hill: Proc. Phys. Soc. London 65 (1952), p. 349

[8] J. D. Eshelby: Proc. Roy. Soc. A 241 (1957), p. 376

[9] E. Kröner: Z. Physik 151 (1958), p. 504

[10] M.van Leeuwen, J.-D. Kamminga and E. J. Mittemeijer: J. Appl. Phys. 86 (1999), p. 1904

[11] J. Stickforth: Tech. Mitt. Krupp - Forsch.-Ber. 24 (1966), p. 89

[12] M. Leoni, U. Welzel, P. Lamparter, E. J. Mittemeijer and J.-D. Kamminga: Phil. Mag. A 81(2001), p. 597

[13] U. Welzel, M. Leoni and E.J. Mittemeijer: Phil. Mag. 83 (2003), p. 603

[14] U. Welzel, M. Leoni and E.J. Mittemeijer in: Diffraction analysis of the microstructure of materials, edited by E.J. Mittemeijer and P. Scardi (Springer, Berlin 2004), pp. 363-390.

[15] J. Krier, H. Ruppersberg, M. Berveiller and P. Lipinski: Textures and Microstructures, 14-18 (1991), p. 1147

[16] J. Krier: Doctorate thesis (Université de Metz, France: 1993).

[17] N. Koch, U. Welzel, H. Wern and E.J. Mittemeijer, accepted for publication in Phil. Mag.

[18] R.W.Vook and F.Witt: J. Appl. Phys. 36 (1965), p. 2169

[19] M. A. Meyers and K. K. Chawla: Mechanical metallurgy, principles and applications (Englewood Cliffs, New Jersey: Prentice-Hall: 1984), p. 57.

[20]U. Welzel, S. Fréour and E.J. Mittemeijer: in preparation 